\title{
Le Web 2.0 dans les bibliothèques : vers un nouveau modèle de service
}

\author{
Web 2.0 in Libraries: Towards a New Model of Service \\ Web 2.0 en las bibliotecas: hacia un nuevo modelo de servicio
}

\section{Houda Bachisse et Christine Dufour}

Volume 57, numéro 1, janvier-mars 2011

URI : https://id.erudit.org/iderudit/1028960ar

DOI : https://doi.org/10.7202/1028960ar

\section{Aller au sommaire du numéro}

\section{Éditeur(s)}

Association pour l'avancement des sciences et des techniques de la documentation (ASTED)

\section{ISSN}

0315-2340 (imprimé)

2291-8949 (numérique)

\section{Découvrir la revue}

\section{Citer cet article}

Bachisse, H. \& Dufour, C. (2011). Le Web 2.0 dans les bibliothèques : vers un nouveau modèle de service. Documentation et bibliothèques, 57(1), 5-17. https://doi.org/10.7202/1028960ar

\section{Résumé de l'article}

Cet article introduit le concept du Web 2.0 pour définir celui de « bibliothèque 2.0 ». Il suggère que les réflexions récentes sur l'évolution de l'offre de service des bibliothèques passent par une connaissance des technologies et de la philosophie qui sous-tendent le Web 2.0. L'objectif d'une telle démarche est d'explorer de nouvelles approches de fonctionnement des bibliothèques qui coïncident avec les nouvelles approches de recherche et d'appropriation de l'information des usagers. Les technologies comme les blogues, les sites Wikis, les fils RSS ou encore les outils de réseautage sociaux jouent un rôle important dans cette évolution de prestation de service. Notre exploration passera entre autres par la présentation d'une nouvelle conceptualisation du fonctionnement des bibliothèques où les notions de co-création, de service évolutif et d'interdépendance constituent les maîtres mots, voire l'échafaudage d'une bibliothèque "écosystème ». En se basant sur la notion de co-création, nous étudierons la place de l'utilisateur dans cet " écosystème " ainsi que les changements à effectuer tant du point de vue des usages, de l'organisation que des missions.
Tous droits réservés (c) Association pour l'avancement des sciences et des techniques de la documentation (ASTED), 2011
Ce document est protégé par la loi sur le droit d'auteur. L'utilisation des services d'Érudit (y compris la reproduction) est assujettie à sa politique d'utilisation que vous pouvez consulter en ligne.

https://apropos.erudit.org/fr/usagers/politique-dutilisation/ 


\title{
Le Web 2.0 dans les bibliothèques : vers un nouveau modèle de service
}

\author{
HOUDA BACHISSE \\ Édimestre, Agence universitaire de la Francophonie (AUF), Montréal \\ houda.bachisse@auf.org
}

\section{ChRISTINE DUfour}

Professeure adjointe, EBSI, Université de Montréal

christine.dufour@umontreal.ca

\section{RÉSUMÉ | ABSTRACTS | RESUMEN}

Cet article introduit le concept du Web 2.0 pour définir celui de "bibliothèque 2.0 ". Il suggère que les réflexions récentes sur l'évolution de l'offre de service des bibliothèques passent par une connaissance des technologies et de la philosophie qui soustendent le Web 2.o. L'objectif d'une telle démarche est d'explorer de nouvelles approches de fonctionnement des bibliothèques qui coincident avec les nouvelles approches de recherche et d'appropriation de l'information des usagers. Les technologies comme les blogues, les sites Wikis, les fils RSS ou encore les outils de réseautage sociaux jouent un rôle important dans cette évolution de prestation de service. Notre exploration passera entre autres par la présentation d'une nouvelle conceptualisation du fonctionnement des bibliothèques où les notions de co-création, de service évolutif et d'interdépendance constituent les maîtres mots, voire l'échafaudage d'une bibliothèque "écosystème». En se basant sur la notion de co-création, nous étudierons la place de l'utilisateur dans cet "écosystème " ainsi que les changements à effectuer tant $d u$ point de vue des usages, de l'organisation que des missions.

Web 2.o in Libraries: Towards a New Model of Service

This article uses the concept of Web 2.0 to define the concept of "library 2.0". It suggests that the recent analyses of the evolution of the service offer of libraries are necessarily linked to the knowledge of technologies and to the philosophy underlying the Web 2.o. The purpose of such an undertaking is to examine new ways of operating libraries that coincide with the research and the informationseeking habits of users. Technologies such as blogs, Wiki sites, RSS feeds and social networking play an important role in the evolution of the service offer. This article examines a new concept of library operations in which notions of co-creation and evolutionary and interdependent service are key; they constitute the framework of an "ecosystem" library. With co-creation in mind, the authors study the role of the user in this "ecosystem" as well as the changes to be undertaken from the point of view of the users, the organisation and its mission.

Web 2.o en las bibliotecas : hacia un nuevo modelo de servicio

Este artículo introduce el concepto de Web 2.o para definir el de " biblioteca 2.0 ". Sugiere que las reflexiones recientes sobre la evolución de la oferta del servicio bibliotecario han desarrollado un conocimiento de las tecnologías y de la filosofía que sirven de base al Web 2.o. El objetivo de este proceso consiste en explorar nuevos acercamientos al funcionamiento de las bibliotecas, que coinciden con los nuevos métodos de búsqueda y apropiación de la información de los usuarios. Las tecnologías como los blogs, los sitios Wiki, los hilos RSS o las herramientas de conexión social desempeñan una función clave en la evolución de este servicio. Nuestra investigación incluirá, entre otras cosas, la presentación de una nueva conceptualización del funcionamiento de las bibliotecas, en la que las nociones de co-creación, de servicio evolutivo y de interdependencia constituyen las palabras clave, es decir, la creación de una biblioteca " ecosistema ". Basándonos en la noción de co-creación, estudiaremos la función del usuario en este " ecosistema ", así como los intercambios que se efectuarán, desde el punto de vista de los usuarios, de la organización y de las misiones.

\section{Introduction}

A VEC L'AVÈNEMENT DU NUMÉRIQUE et de la multitude de services proposés, notamment avec le Web 2.0, les usagers des bibliothèques sont devenus plus autonomes aussi bien dans leurs démarches de recherche que dans leurs processus d'appropriation de l'information. Ils dessaisissent ainsi les bibliothèques de l'une des prestations qu'elles accomplissaient avec excellence, la médiation. Face à cette évolution, les bibliothèques doivent s'interroger quant à leurs missions et leurs services pour mieux s'adapter et adopter ces nouvelles réalités. Ainsi, le présent article se propose d'explorer, par le truchement des écrits sur le sujet, une nouvelle approche de service pour les bibliothèques où il est question de concilier les paradigmes traditionnels de la bibliothéconomie aux tendances modernes du Web 2.0. Une fois le concept de Web 2.0 défini, celui de la bibliothèque 2.0 est examiné afin de proposer un modèle de service novateur basé sur une démarche proactive, créative et collaborative.

\section{Des bibliothèques en évolution}

"Désertées", voilà le terme qui revient fréquemment et de plus en plus dans les travaux de certains chercheurs du domaine des sciences de l'information lorsqu'ils évoquent la situation actuelle des bibliothèques face à l'hégémonie du numérique (Martell, 2005; Albanese, 2003 ; Carlson, 2001). D’autres, interpellés par la même situation, s'interrogent sur l'avenir de ce qu'on appelait dans le passé le temple du savoir. Ils se demandent si les documents imprimés, au même titre que les bâtiments des bibliothèques, ne se réduiraient plus qu'à de simples artefacts de musées (Weise, 


\section{La bibliothèque s'estompe sur le radar des usagers qui préfèrent la contourner en se rabattant sur d'autres sources informationnelles, provoquant ainsi une baisse considérable de fréquentation.}

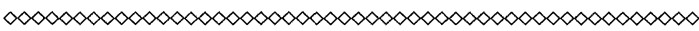

2004). L'avènement du document numérique et la prolifération des sources d'information alternatives mettent les bibliothèques dans un environnement compétitif (Campbell, 2006: 16; Borgman, 2003: 653) altérant ainsi les orientations qui ont été données à ces institutions. Ross \& Sennyey (2008: 145) parlent de perte de captivité des utilisateurs. Pour ces deux auteurs, la bibliothèque s'estompe sur le radar des usagers qui préfèrent la contourner en se rabattant sur d'autres sources informationnelles, provoquant ainsi une baisse considérable de fréquentation.

Les bibliothèques universitaires québécoises n'échappent pas à ce phénomène, comme en témoignent les travaux du sous-comité des bibliothèques de la Conférence des recteurs et des principaux des universités du Québec (CREPUQ). Le nombre d'entrées dans les bibliothèques universitaires (BU), à titre d'exemple, a accusé une forte baisse entre 1992 et 2007. En 1991-1992, on dénombrait près de 90 visites par personne dans l'année alors qu'en 2006-2007, ce nombre n'était plus que de 67 visites, soit une diminution de presque $26 \%$. La consultation des documents sur place accuse également une forte baisse de $42,1 \%$, passant de 61,2 documents par personne en 1991-1992 à 19,1 en 2006-2007 (CREPUQ, 2008). Ces chiffres sont d'autant plus alarmants que le nombre d'étudiants en équivalent à temps complet par place assise a augmenté de 1,2 \% dans les universités, passant de 7,7 en 1991-1992 à 8,9 en 20062007 (CREPUQ, 2008).

Les nouvelles réalités numériques sont en partie la cause des transformations que connaissent actuellement les bibliothèques. La CREPUQ, par exemple, explique les changements dans la fréquentation et l'utilisation des BU par l'accès à distance aux sources informationnelles. La nature même de l'information a changé (format, modalités de création, modèles de diffusion, etc.) ; partout elle est devenue multiple, diffuse et accessible sous des formes diverses et variées. Les besoins et les attentes des utilisateurs ont aussi évolué. Ces derniers adoptent aujourd'hui des comportements informationnels différents provoqués par le déploiement d'Internet et la banalisation des services Web. Ces comportements se manifestent dans les façons de communiquer, d'interagir, d'acquérir et de partager les connaissances. Wilson affirme :

"The nature of access to information is changing rapidly and changing radically. The source of the change is well known-it is the emergence of the Internet and the transformative character of the World Wide Web technology, which, quite suddenly, has changed the ground rules for the production of and access to scholarly communication $»(1998: 15)$.

Cette nouvelle réalité perturbe le modèle traditionnel des bibliothèques et en questionne l'efficience, les poussant à moderniser leurs offres de service (Campbell, 2006 : 20 ; Wilson, 1998 : 16). Cette modernisation altère la notion d'espace et de service : on parle désormais de bibliothèques hors les murs, extensions logiques des bibliothèques classiques (Holmberg et al., 2009). En plus des services traditionnels, ces institutions hors les murs fournissent des contenus et d'autres types de services qui impliquent davantage les usagers et qui ne peuvent être délivrés qu'à travers le Web. Ce changement radical est qualifié dans le milieu bibliothéconomique par l'expression «bibliothèque 2.0 ». Il caractérise à la fois une évolution dans les services, puisqu'il intègre désormais la technologie participative, et une évolution dans les pratiques, puisqu'il s'agit de reconsidérer la position des usagers dans cette offre de service. Afin d'appréhender le concept de bibliothèque 2.0, nous commencerons dans la section suivante par nous intéresser au phénomène qui est à la base de l'apparition de ce concept : le Web 2.0.

\section{Le Web 2.0}

Révolution pour les uns, simple évolution pour les autres, le Web 2.0 nourrit les polémiques entre les acteurs du Web et ses observateurs (Gervais, 2007:3). Cette entité, aux contours parfois flous (Quoniam \& Boutet, 2008 : 133), est au cœur des discussions entre les spécialistes du Web depuis son avènement en 2004, et son acception ne fait toujours pas l'unanimité (Curran et al., 2006: 47). Certains voient en lui un mythe ou une hyperbole (Breeding, 2007). D'autres distinguent, au-delà de son effet de mode, un ensemble d'outils en évolution dont les citoyens peuvent bénéficier en ligne (Stephens, 2007: 9). Afin de réussir à mieux définir les contours du Web 2.0, nous l'examinerons sous les différents angles et dimensions sous lesquels il a été abordé.

\section{Le Web 2.0 : les principes fondamentaux}

L'essence du Web 2.0 repose sur deux principes fondamentaux. Le premier principe, édicté par O'Reilly (2007), fondateur et figure de proue du Web 2.0, est que le Web 2.0 représente une plateforme informatique à part entière, et pas seulement une collection de sites Web isolés. Ainsi, les applications ne sont plus installées sur les ordinateurs personnels, mais plutôt livrées et utilisées sur le Web sous forme de services (Kroski, 2008: 3). Le réseau devient ainsi une plateforme que 
personne ne possède réellement (Evans, 2007: 3). Il s'avère alors être aussi efficace qu'un logiciel et c'est ce qui fait la particularité du Web 2.0: les utilisateurs réalisent enfin que ce n'est pas le logiciel qui fait le Web, mais bien les services (O'Reilly, $2007: 19$ ).

Le deuxième principe fondamental qui caractérise le Web 2.0 est celui de l'intelligence collective, qui lui donne toute sa puissance. On peut définir l'intelligence collective comme "une intelligence partout distribuée, sans cesse valorisée, coordonnée en temps réel, qui aboutit à une mobilisation effective des compétences " (Levy, 2006: 29). Cette mobilisation collective des compétences permet, selon Surowiecki, de profiter de l'intelligence et du savoir-faire d'un grand nombre de personnes, la foule se révélant bien plus intelligente et efficace que l'individu, aussi brillant soit-il (2004:31). C'est ce que l'on appelle "la sagesse des foules" (Wisdom of Crowds). D'ailleurs, le succès de certains produits, comme l'encyclopédie universelle Wikipédia, dont l'alimentation est la responsabilité des internautes, prend sa source dans l'exploitation de cette sagesse des foules. Les produits prennent ainsi appui sur un approvisionnement de contenu par la foule (crowdsourcing), utilisant la créativité, l'intelligence et le savoir-faire d'un grand nombre d'internautes, à moindres coûts (Allard, $2007: 23$ ). C'est sur cette philosophie de participation et de partage que repose le Web 2.o. Il est dit alors participatif, collaboratif à forte dimension sociale (Stephens, 2007 : 10 ; Kroski, 2007 : 2).

\section{Le Web 2.0 : la dimension sociale}

«Web participatif» et «Web collaboratif» sont deux synonymes très utilisés pour décrire le Web 2.o. Ce nouveau Web est basé sur des systèmes architecturaux intelligents qui permettent aux utilisateurs de collaborer et de contribuer au développement et à l'extension du contenu numérique (O’Reilly, 2007 ; Japon. Ministry of Internal Affairs and Communication (MIC), 2006; OCDE, 2006a, 2006b). L'engagement des utilisateurs apporte une valeur ajoutée au Web (Liu, 2008: 10), ce qui contribue à bâtir un capital social (Evans, 2007:5) : plus les usagers sont nombreux, plus la valeur des services Web est grande. En fait, dans le Web 2.0, il y a une volonté de donner le pouvoir aux internautes pour qu'ils deviennent à la fois acteurs, auteurs et lecteurs, bref, des collaborateurs et non plus de simples consommateurs (I-expo, 2007 : 249). Pour Quoniam et Boutet (2008: 134), le Web 2.0 caractérise la révolution connectique qui marque l'avènement du "Web écrit » (Writable Web), appelé aussi Web contributif, un Web où les individus ont le droit d'écriture ${ }^{1}$ sur le contenu et donc le droit de participer à son enrichissement. Ils prennent ainsi le contrôle de cet outil qui leur était initialement

1. En informatique il existe trois droits de base : lecture, écriture et exécution d'un fichier.
Les développeurs exploitent le

caractère collaboratif $d u$ Web 2.0 pour

produire de meilleures applications, simples, efficientes et en adéquation avec les besoins des utilisateurs.

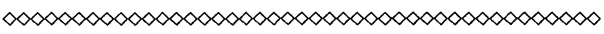

destiné. C'est un nouveau mode d'interaction, voire de socialisation, qui dépasse la dimension technologique pour embrasser la dimension humaine (Anderson, 2007 ; Grossman, 2007 ; Deshpande \& Jadad, 2006).

Les changements apportés par le Web 2.0 ne sont pas que sociaux. Le Web 2.0 apporte aussi de nouveaux outils aux internautes. Certains auteurs adoptent une perspective technologique pour le définir et le décrire.

\section{Le Web 2.0 : la dimension technologique}

Le Web 2.o est une plateforme qui, d'un point de vue technologique :

- utilise de nouvelles techniques simples de publication ;

- repose sur des interfaces plus conviviales;

- et se base sur des protocoles de communication plus ouverts (Bernat \& Mesguich, 2006).

C'est un Web qui intègre de multiples technologies afin de devenir véritablement interactif (ergonomie des sites Web et interfaces utilisateurs, feuilles de style CSS, syndication de contenu, technologies $\mathrm{Ajax}^{2}$, etc.).

Ce Web de la nouvelle génération se caractérise, entre autres, par le développement du haut débit et la généralisation des équipements informatiques (NemecPoncik, 2007), mais aussi par l'appartenance au mouvement du logiciel libre (Gervais, 2007).

Les développeurs exploitent le caractère collaboratif du Web 2.0 pour produire de meilleures applications, simples, efficientes et en adéquation avec les besoins des utilisateurs. Les technologies ainsi développées sont alors partagées et servent à en construire de nouvelles (Kroski : 2008). Du point de vue des spécialistes du design, le Web 2.0 est un moyen efficace d'améliorer l'ergonomie des sites Web et de perfectionner le style (Quoniam \& Boutet, 2008 : 134).

Le Web 2.0 incarne une série de changements difficiles à résumer en une seule approche. Toutefois, un certain consensus se dégage pour considérer que le Web 2.0 marque le passage d'un Web classique vers une nouvelle étape qui, sur le plan humain, fait place à des internautes plus actifs, plus impliqués, avec plus de

2. Asynchronous JavaScript And XML (AJAX) est une combinaison de technologies qui permet de charger le contenu d'une partie de page Web sans pour autant avoir à recharger la page entière. On charge ainsi uniquement les éléments dont on a besoin (Kroski, 2008: 5; Black, 2007 : 3). 
Comme pour les partisans du Web 2.0, pour qui ce phénomène symbolise une réelle évolution $d u$ Web, les adeptes de la bibliothèque 2.0 affirment que cette dernière représente une évolution majeure dans l'histoire des bibliothèques.

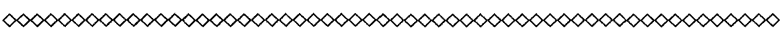

pouvoir et qui, sur le plan technologique, interpelle des applications plus mutualisées, ergonomiques, ouvertes et intelligentes.

Plusieurs organisations tentent de tirer profit du potentiel du Web 2.0. Elles l'utilisent non seulement comme levier de transformation, mais aussi comme moyen d'innovation. Les bibliothèques, à l'instar de ces organisations soucieuses de leur avenir, doivent utiliser à bon escient les outils du Web 2.o. Elles doivent non seulement les adopter, mais également se les approprier pour perfectionner et affiner leur prestation de services sans pour autant la changer fondamentalement. Plusieurs auteurs utilisent l'expression "bibliothèque 2.0 " pour marquer ce changement dans le modèle de service des bibliothèques (Holemberg et al., 2009; Shoniwa \& Hall, 2008; Curran et al., 2006; Maness, 2006 ; Casey \& Savastinuk, 2006 ; Houghton, 2005 ; Miller \& Chad, 2005).

\section{Le Web 2.0 dans les bibliothèques : la bibliothèque 2.0}

Le concept de «bibliothèque 2.0 " est apparu en 2005. Il a été introduit par Casey, directeur de la division Technologie de l'information de la Gwinnett County Public Library d'Atlanta, dans son blogue Library Crunch (<http://www.librarycrunch.com $>$ ) pour signifier que tous les services proposés par le Web 2.0 sont applicables au domaine des bibliothèques $(\mathrm{Xu}$ et al., 2009; Maness, 2006). Pour Casey, les bibliothécaires doivent changer la manière dont ils conçoivent et livrent leurs services. Le principal changement qui caractérise la bibliothèque 2.0 est la manière d'interagir avec les usagers. Ces derniers, par leurs nouveaux comportements informationnels, poussent les bibliothèques à reconsidérer leurs offres de services de façon à les impliquer davantage. Ainsi, les technologies du Web 2.o joueraient un rôle significatif dans le cadre de cette évolution (Casey \& Savastinuk, 2006, cité par Crawford, 2006).

On remarque dans la littérature un manque de consensus sur la définition de "bibliothèque 2.0 " (Holmberg et al., 2009, 670; Shoniwa \& Hall, 2008, 69). Plusieurs ont essayé de l'expliciter et d'explorer ses limites et continuent d'ailleurs de le faire, mais malgré cela, il n'existe toujours pas de définition normative de ce concept. La bibliothèque 2.0 est perçue de différentes manières : un vrai changement de paradigme pour les uns (Xu et al., 2009: 324 ; Farkas, 2007), une nouvelle philosophie pour les autres (Casey \& Savastinuk, 2006, cité par Crawford, 2006), un phénomène orienté technologies pour certains (Maness, 2006), une idéologie motivée par les services pour d'autres (Casey \& Savastinuk, 2006). Les points de vue oscillent entre les adeptes et les sceptiques du concept de bibliothèque 2.0.

\section{La bibliothèque 2.0 : les adeptes}

Comme pour les partisans du Web 2.0, pour qui ce phénomène symbolise une réelle évolution du Web, les adeptes de la bibliothèque 2.0 affirment que cette dernière représente une évolution majeure dans l'histoire des bibliothèques. Les plus fervents d'entre eux affirment que le terme bibliothèque 2.0, bien qu'il soit a priori conflictuel, présuppose que tous les services rendus par les bibliothèques sont obsolètes et nécessitent d'être renouvelés au regard des nouveautés apportées par cette nouvelle génération du Web. Une large majorité des adeptes de la bibliothèque 2.0 la définissent selon les mêmes deux perspectives utilisées pour définir le Web 2.0: la perspective sociale et la perspective techniciste.

\section{Perspective sociale}

On peut examiner la bibliothèque $2.0 \mathrm{du}$ point de vue de ses utilisateurs et de l'offre de services qui leur est faite. Casey \& Savastinuk (2006) affirment que c'est une obligation pour les bibliothèques de revitaliser leur façon d'interagir avec les usagers en adoptant les technologies du Web 2.o. L'objectif premier serait d'améliorer les moyens de communication avec les usagers réels et d'attirer les non-usagers. Casey \& Savastinuk (2006) présentent la bibliothèque 2.0 comme un nouveau modèle qui inclut l'utilisateur dans la mise en œuvre des services de la bibliothèque. C'est un modèle de service qui encourage le changement, au cœur duquel se trouve l'usager. Plus qu'un changement de modèle, c'est un changement de philosophie de service construite sur trois éléments selon Casey (2006) :

«Library 2.0 is, to me, a service philosophy built upon three things : 1) a willingness to change and try new things; 2) a willingness to constantly re-evaluate our service offerings; and finally, 3) a willingness to look outside our own world for solutions, be they technology-driven or not " (cité par Crawford : 2006, 5).

Ainsi, les services offerts par les bibliothèques doivent être en lien direct avec les besoins des usagers d'aujourd'hui, grâce notamment à l'utilisation de technologies favorisant l'interaction et la collaboration (Houghton, 2005; Curran et al., 2006). La bibliothèque ne se positionne plus comme un "sanctuaire 
du savoir», expression qui a toujours accompagné la définition des bibliothèques à ce jour, mais comme un acteur social dont l'objectif est d'être au service de la communauté des utilisateurs et dont les prestations sont dictées par leurs besoins (Houghton, 2005). Les utilisateurs détiendraient ainsi le contrôle sur la prestation des services qu'ils désirent dans cette évolution naturelle qu'est la bibliothèque 2.0 (Brevick, 2006).

Jusque-là, les bibliothèques étaient considérées comme la source d'autorité incontournable pour les usagers (Campbell, 2006: 17). L'imprimé incarnait cette autorité. Le passage vers la philosophie du Web 2.0 marque le déplacement de l'autorité de l'institution vers celle des usagers, considérés désormais comme des collaborateurs. On ne parle plus de culture d'autorité, mais plutôt d'une culture de confiance totale (radical trust) en l'usager et de partage, nouvelle dimension dans le mandat d'exercice des bibliothèques (Fichter, 2006).

On voit ainsi un certain consensus émerger chez les adeptes quant à la bibliothèque 2.0, bien qu'elle soit abordée sous différents angles :

- la bibliothèque 2.0 incarnerait un nouveau modèle de modernisation des services des bibliothèques ;

- elle illustrerait la prise de pouvoir des usagers, désormais impliqués dans cette offre de service;

- elle représenterait presqu'une obligation pour les bibliothèques qui, pour innover et attirer les non-usagers, n'ont d'autres choix que de s'inscrire dans la nouvelle culture informationnelle des utilisateurs.

\section{Perspective techniciste}

La bibliothèque 2.0 peut aussi être considérée sous l'angle des différents outils issus du Web 2.o qu'elle peut exploiter. En effet, plusieurs auteurs présentent la bibliothèque 2.0 comme le fruit de l'implémentation des technologies du Web 2.o dans l'environnement des bibliothèques. On la décrit même comme une résultante, voire un prolongement naturel du phénomène global du Web 2.0, qui semble avoir des implications sur la façon dont les bibliothèques et les bibliothécaires travaillent, collaborent et livrent leurs services (Connor, $2007: 2$ ).

À titre d'exemple, Maness (2006) propose que cette définition de la bibliothèque 2.0 , centrée sur les technologies, soit adoptée par la communauté des spécialistes en bibliothéconomie : "Library 2.0 is the application of interactive, collaborative, and multi-media Web-based technologies to Web-based library services and collections" (Maness, 2006).

La bibliothèque 2.0 serait bâtie sur les principes du Web 2.0, des principes qui supposent que les services et les données qui étaient disponibles uniquement pour lecture peuvent être réadaptés et réutilisés de façon à créer des applications composites (Curran et al., 2006 : 47).

Bien que ses adeptes soient nombreux, la bibliothèque 2.0 n'est pas sans avoir ses propres critiques.

\section{La bibliothèque 2.0 : les sceptiques}

Comme pour les sceptiques du Web 2.0, pour qui ce dernier n'incarnerait qu'une nouvelle étape dans l'histoire du Web et non une véritable révolution, la bibliothèque 2.0 a ses détracteurs. Certains restent sceptiques face au concept qui, en soi, n'a pas de réelle signification; il n'apporterait selon eux rien de nouveau au discours des professionnels de l'information. Les bibliothèques et les bibliothécaires ont toujours été au diapason des nouvelles tendances. La technologie a toujours été présente dans les prestations de services de ces institutions. Toutefois, cette présence n'a jamais chamboulé radicalement le mandat de service de ces dernières (Plutchak, 2006, cité par Crawford, 2006 : 29).

L'usager a constamment été au centre des préoccupations des bibliothèques. Ces dernières ont toujours fait face à ses exigences et n'ont cessé de s'adapter à ses besoins ainsi qu'aux tendances du moment. Qu'il s'agisse de la première génération des bibliothèques ou de la seconde, l'approche est fondamentalement la même. Il ne s'agit donc que d'une simple progression dans la nomenclature utilisée pour désigner ce changement (Dowling, 2006, cité par Crawford, 2006: 30). Cohen (2006) fait également partie de ceux qui jugent que la bibliothèque 2.0 est un concept qui ne mérite pas toute cette attention, le terme n'apportant aucun renouveau théorique à la bibliothéconomie traditionnelle (Cohen, 2006, cité par Crawford, 2006: 28).

\section{La bibliothèque 2.0 : essai de définition}

Les sections précédentes ont démontré que le concept de «bibliothèque 2.0 " ne fait pas l'unanimité, même parmi ses adeptes. De nombreuses définitions existent qui la présentent tantôt sous la perspective sociale, tantôt sous l'angle technologique. Malgré cette diversité et cette absence de consensus, l'examen de différentes définitions permet de faire ressortir sept composantes fondamentales de la bibliothèque 2.0 (Holmberg et al., 20093) : l'interactivité, les utilisateurs, la participation, les bibliothèques et les services de bibliothèque, le Web et le Web 2.0, les aspects sociaux ainsi que la technologie et les outils.

Plus précisément, l'examen des différentes visions et analyses sous lesquelles le vocable a été abordé permet de dégager les caractéristiques d'une bibliothèque 2.0.

3. Holmberg et al. (2009), par le biais d'une étude qualitative, ont recueilli et analysé des définitions fournies par 29 participants (praticiens et chercheurs) lors d'entrevues pour en faire ressortir les composantes principales. 
En résumé, la bibliothèque 2.0 se base sur la collaboration mutuelle, sur le service évolutif, sur la réciprocité et l'interdépendance.

$\infty<\infty<\infty<\infty<\infty<\infty<\infty<\infty<\infty<\infty<\infty<\infty<\infty<\infty<\infty<\infty<\infty<\infty<\infty$

Ainsi, la bibliothèque 2.0 est :

- une entité physique et multi-ressources qui offre aux usagers l'information dont ils ont besoin et sous la forme qu'ils désirent (collections imprimées, collections numériques, collections multimédias, etc.) ;

- une entité virtuelle, présente, voire omniprésente, prête à servir ses usagers et à leur offrir un accès à l'information, peu importe où ils se trouvent et à quel moment ils veulent $\mathrm{y}$ accéder ;

- une bibliothèque très proche des utilisateurs qui maîtrise les outils qu'utilisent ses usagers afin de leur offrir des services via ces mêmes outils, et afin d'attirer les non-usagers ;

- une entité innovante à forte valeur ajoutée qui suit la mouvance de ses usagers tout en essayant d'apporter une plus grande valeur ajoutée par rapport au modèle traditionnel ;

- une bibliothèque qui va chercher à investir de nouveaux territoires en exploitant notamment le potentiel de la longue traîne. Naviguer dans la longue traîne consiste à recourir aux ressources d'autres bibliothèques (par ex. : les prêts interbibliothèques), à considérer les recommandations et les critiques de livres faites par des utilisateurs, à se procurer des ouvrages chez des détaillants de livres d'occasion, bref cela consiste à aller là où les utilisateurs ne peuvent pas aller, en localisant et en évaluant les meilleures ressources disponibles (Mossman, 2006) ;

- une entité ouverte. L'ouverture se manifeste au niveau de sa relation avec les usagers, laquelle n'est plus fondée sur l'autorité, mais sur la confiance mutuelle. Les usagers sont invités à collaborer avec la bibliothèque, en alimentant son contenu, en le partageant et en l'indexant, bref en participant à son évolution.

En résumé, la bibliothèque 2.0 se base sur la collaboration mutuelle, sur le service évolutif, sur la réciprocité et l'interdépendance. On peut ainsi la considérer comme un écosystème4, puisqu'elle établit un lien étroit d'interdépendance avec ses usagers. Une biblio-

4. On entend par écosystème un milieu défini à l'intérieur duquel des organismes vivants interagissent dans une relation d'étroite interdépendance pour former une unité fonctionnelle (Duquet, 1993: 43). Les éléments constituant un écosystème développent un réseau d'interdépendances permettant le maintien et le développement de la vie. thèque sans les usagers qui la fréquentent, qui utilisent ses services, qui l'évaluent constamment et qui contribuent à son évolution, est une entité vouée à disparaître. Au même titre que les bibliothèques, des usagers laissés pour compte, sans orientation, sans médiation, sans gouvernail pour ne pas s'égarer dans la masse informationnelle, sont des usagers mal informés, voire perdus.

La bibliothèque 2.0 représente un changement fondamental dans la manière dont les bibliothèques construiront et livreront leurs services dans le futur. La bibliothèque 2.0 n'est pas une fin en soi, mais elle représente une étape cruciale dans l'histoire des bibliothèques en marquant une transformation de leur mode de service. Ce changement passe par une nouvelle approche et un nouveau modèle de service.

\section{La bibliothèque 2.0 : un nouveau modèle de service}

Le nouveau modèle de service de la bibliothèque 2.0 repose sur quatre principes fondamentaux :

- Se promouvoir ;

- S'ouvrir à l'usager ;

- Améliorer les outils existants et délivrer de nouveaux services ;

- Se poser en forum social.

\section{Se promouvoir}

L'approche marketing 5 n'est pas une démarche nouvelle pour les bibliothèques. C'est une réflexion qui a été entamée voilà plusieurs années déjà par différents chercheurs (Ibnlkhyat, 2005 ; Salaün \& Muet, 2001). En a résulté un cadre d'analyse et d'intervention particulièrement important dans le contexte de transformation intense auquel les bibliothèques sont constamment confrontées. Ce cadre leur a permis d'être plus à l'écoute des attentes des usagers ainsi que de les servir de façon plus ciblée. La bibliothèque 2.0 s'inscrit totalement dans ce cadre. En effet, les bibliothèques doivent se repositionner dans un environnement numérique qui a évolué et a modifié le comportement des usagers. N'ayant plus le quasi-monopole de l'offre informationnelle, la bibliothèque se doit d'être omniprésente et ouverte sur l'extérieur.

\section{Une bibliothèque omniprésente}

Les services de la bibliothèque doivent être offerts dans l'environnement des usagers et selon leur propre logique (Gordon, 2006: 52 ; Miller \& Chad, 2005). Ils doivent être accessibles à partir d'un large éventail de

\footnotetext{
5. Par définition, le marketing est l'ensemble des démarches qui concourent à la création, à la conservation et à l'élargissement de la clientèle de l'entreprise (Lendrevie \& Lindon, 2000: 4)
} 
dispositifs et intégrés avec ceux qui sont offerts déjà par certains services Web, et ce, en tout temps et peu importe les lieux où se trouvent les usagers. Les bibliothèques doivent devenir "omniprésentes" ("pervasive library»), sans se limiter uniquement aux services rendus physiquement, ni aux catalogues en ligne. Elles doivent ainsi offrir un ensemble d'autres services comme les portails documentaires, les bureaux virtuels ou les espaces de commerce électronique, etc. (Miller \& Chad, 2005: 9), exploitant ainsi, à bon escient, les nouvelles technologies.

Farkas (2007) partage ce point de vue en évoquant la notion d'ubiquité comme principe fondamental de la bibliothèque 2.0. La bibliothèque doit être moins présente physiquement, mais omniprésente, plus humaine, plus orientée vers les usagers, moins concentrée sur elle-même. La survie de la bibliothèque dépend de la manière dont elle s'adapte aux pratiques des usagers (Curran et al., 2007). Ceci est particulièrement vrai dans le contexte des bibliothèques universitaires, dont les usagers préfèrent accéder à l'information à distance via Internet (CREPUQ, 2008).

\section{Une bibliothèque sans frontières}

Les bibliothèques doivent être au cœur du processus de démocratisation de l'information. Elles doivent œuvrer pour une meilleure visibilité des services et pour un désenclavement des espaces documentaires. Pour ce faire, les ressources des bibliothèques doivent être disponibles à tout moment, sans obstacles à leur utilisation ni à leur réutilisation. Elles doivent être davantage visibles sur le Web et pouvoir être récupérées par les moteurs de recherche au lieu de rester "cachées" dans des catalogues ou conservées dans des bases de données propriétaires qui ne sont accessibles que par le site Web de la bibliothèque (Miller \& Chad, 2005).

\section{S'ouvrir à l'usager}

La philosophie de la bibliothèque 2.0 doit reposer sur un modèle de service qui invite les utilisateurs à participer à la vie de la bibliothèque et à la personnalisation des services qu'ils désirent recevoir, et leur donner les moyens pour y arriver. Les bibliothèques doivent encourager le développement d'une culture facilitant la participation et la collaboration entre les différents acteurs associés aux bibliothèques (Miller \& Chad, 2005). Elles doivent adopter une approche proactive qui consiste à aller vers l'usager et à construire avec lui. Ce dernier ne doit plus être considéré comme simple usager final, mais comme un acteur-collaborateur qui participe à la vie de la bibliothèque, à son développement et à son innovation. On parle d'une approche "centrée usager" (user centric). Ces nouveaux services des bibliothèques vont devoir être "domestiqués " par les usagers qui passent de la perception d'espaces de
La bibliothèque doit être moins

présente physiquement, mais omniprésente, plus humaine, plus

orientée vers les usagers, moins

concentrée sur elle-même.

$\infty<\infty<\infty<\infty<\infty<\infty<\infty<\infty<\infty<\infty<\infty<\infty<\infty<\infty<\infty<$

travail à celle de lieu de socialisation, de conversation et d'échanges (Bennett, 2005 : 22).

Plusieurs moyens sont à la disposition des gestionnaires et des bibliothécaires pour s'ouvrir à cette nouvelle génération d'usagers et pour les inviter à participer. Casey \& Savastinuk (2006) proposent d'explorer la théorie de la "longue traîne », empruntée au monde du commerce et de la distribution, pour interagir avec les usagers. Cette théorie incarne le passage du marché de masse vers un marché composé de plusieurs niches où le choix des consommateurs se répartit sur des multitudes de produits (Anderson, 2007). En considérant cette théorie, la bibliothèque sera en mesure d'atteindre un large éventail d'utilisateurs en leur offrant les produits qui présentent un intérêt particulier pour eux. D'autres auteurs (Linh, 2008; Shoniwa \& Hall, 2007; Mathews, 2007; Sund, 2007; Bradley, 2007; Maness, 2006 ; Stephens, 2006 ; Clyde, 2004) appellent à exploiter le potentiel des technologies du Web 2.o par lesquelles les usagers peuvent dorénavant donner leurs avis, expliciter leurs habitudes d'usage, exprimer leurs incompréhensions ou dévoiler d'éventuels dysfonctionnements au sein de la bibliothèque.

\section{Améliorer les outils existants et livrer de nouveaux services}

Jusqu'à aujourd'hui, les bibliothèques ont bien su accomplir la mission pratique que la société leur a confiée : acquérir, organiser, mettre à la disposition du public et conserver les sources disponibles sous quelque forme que ce soit, de sorte que celles-ci soient accessibles et puissent être utilisées. La mise en ligne des catalogues des bibliothèques, puis des collections numérisées incluant les périodiques, les ouvrages ou les autres documents sont quelques exemples des évolutions qu'ont connues les bibliothèques (Accart, 2008: 4). Toutefois, ces exemples incarnent plus une volonté de s'adapter aux changements technologiques qu'un besoin d'innover et d'apporter une certaine valeur ajoutée par rapport à ce qui existe déjà dans l'espace informationnel. En s'associant aux technologies du Web 2.0, les bibliothèques vont pouvoir améliorer leur expertise et se distinguer ainsi des sources alternatives qui les concurrencent. Leur défi est de continuer de répondre à leur mission, de l'approfondir et de la diversifier pour faire face aux nouveaux enjeux de l'organisation et de la 


\section{Les bibliothèques seront considérées ainsi} comme des centres de connaissances locales, une sorte de plateforme pour le stockage et la diffusion de savoirs des communautés locales.

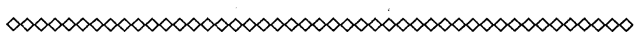

diffusion des ressources numériques. Bien que la littérature ne soit pas unanime sur les démarches à entreprendre par les bibliothèques pour approfondir leurs services traditionnels et les diversifier, certains travaux proposent des démarches qui se recoupent sur plusieurs points (voir par exemple: Curran et al., 2007; Curran et al., 2006 ; Casey \& Savastinuk, 2006 ; Miller \& Chad, 2005). Ainsi, les bibliothèques doivent-elles :

- chercher continuellement de nouvelles opportunités pour diffuser leur offre de service sur de nouveaux canaux et auprès de nouvelles clientèles pour augmenter leur visibilité et montrer leurs richesses et leurs compétences ;

- offrir un site Internet modulable et personnalisable qui a recours à une large gamme de technologies stables qui garantissent l'interopérabilité et la flexibilité pour favoriser cette visibilité ;

- aller vers l'usager en variant les moyens d'accès à la bibliothèque plutôt qu'inciter les usagers à venir au catalogue ;

- proposer des fonctionnalités permettant d'exploiter le contenu à partir d'applications extérieures (fils RSS, moteur de recherche dans ses ressources intégrables à un espace MyGoogle ou Netvibes, etc.);

- contextualiser la bibliothèque de façon à ce qu'elle apparaisse dans le contexte de sites commerciaux comme Amazon (<http://www.amazon.com>) et de services Web 2.0 comme Librarything ${ }^{6}$ ou Google recherche de livres (books. google.fr) (Mercier, 2006) ;

- promouvoir d'autres services, en sus des services traditionnels, tels le prêt et la consultation sur place, pour répondre aux attentes des usagers en ce qui concerne l'accès aux documents électroniques ou à des bases de données, le chargement d'un livre au format $\mathrm{MP}_{3}$, l'écoute ou le chargement d'un balado, etc. ;

- prendre en considération les contributions et commentaires des usagers pour créer et enrichir un environnement documentaire basé sur l'esprit de partage de l'information ;

- construire des représentations de l'information et autres interfaces innovantes (appli-

6. Librarything est une application Web de catalogage social destinée à enregistrer et partager des bibliothèques personnelles et des listes de livres. cations composites) pour enrichir le service documentaire.

\section{Se positionner en forum social}

Maness (2006) présente la bibliothèque 2.0 comme une nouvelle bibliothèque centrée sur l'utilisateur, qui fournit des matériaux multimédias, qui est socialement riche et surtout qui utilise sa communauté pour innover. Les communautés d'usagers sont considérées dans cette nouvelle optique comme des partenaires disposant de connaissances à partager, mais aussi d'une grande liberté qu'il faut modérer. La question qui se pose ici est : comment la bibliothèque peut-elle intervenir et faire partie de la communauté des usagers qu'elle dessert?

Chowdhury et al. (2006) répondent à cette question en proposant que la bibliothèque œuvre pour créer une communauté d'utilisateurs autour d'elle en leur offrant un espace public de communication. Les bibliothèques seront considérées ainsi comme des centres de connaissances locales, une sorte de plateforme pour le stockage et la diffusion de savoirs des communautés locales. Grâce à cette démarche, les bibliothèques garderaient ainsi le contrôle de la description des contenus et la possibilité de les modérer.

\section{La bibliothèque 2.0 : l'intégration des outils du Web 2.0 en support au nouveau modèle de service}

La majorité des auteurs s'accordent à dire que les outils du Web 2.0, qui peuvent renforcer voire améliorer les services des bibliothèques, sont les blogues, les sites Wikis, les fils RSS, les balados, les applications composites, les folksonomies, les plateformes de partage de ressources ainsi que les outils de réseautage social (Linh, 2008; Shoniwa \& Hall, 2007; Bradley, 2007; Maness, 2006 ; Stephens, 2006). L'utilisation de ces technologies permettrait aux bibliothèques d'être en phase avec les comportements de leurs utilisateurs :

"Our thinking behind this is to reach users in their preferred methods of communication, and in fact, the site statistics on our podcasts are high. With blogging, our primary motivation was giving our students a place to reply to us as their comments are important. I think that social networking can encompass anything that reaches out to our patrons in ways that acknowledge them as partners in search for information - we don't want to dictate what we think they need but respect them and their informationseeking behavior by making an attempt to meet them where they are, be that podcasts, blogs, gaming, MySpace pages, presence in Facebook, etc. » (Topper, $2007: 379$ ). 


\section{Les blogues}

Le terme «blogue » est une contraction de « Web " et « $\log$ » qui a été forgée par Jorn Barger en 1997. Elle réfère à une simple page Web composée de brefs paragraphes d'opinions, d'informations personnelles ou de liens appelés billets (posts), classés par ordre antéchronologique dans le style d'un journal personnel en ligne (Kroski, 2008 : 13; Anderson, 2007; Le Meur \& Beauvais, 2007: 1). Les blogues représentent l'une des technologies du Web 2.0 à fort potentiel pour les bibliothèques afin de leur permettre d'accomplir, d'améliorer, voire de moderniser leur offre de service. Comme le précise Clyde (2004), les blogues et les bibliothèques forment une alliance naturelle. Cette alliance est à l'origine d'une nouvelle dynamique de service qui assure une meilleure communication avec les usagers (Stephens, 2006; Clyde, 2004), des services plus interactifs et plus centrés sur les utilisateurs (Maness, 2006) et la dissémination de l'information et des connaissances. En fait, les blogues sont le meilleur moyen de sonder les usagers sur certains aspects de la bibliothèque et donc le moyen d'instaurer une culture de transparence (Stephens, 2006). Par ailleurs, force est de constater que les micro-blogues tel Twitter gagnent en popularité, notamment dans les bibliothèques universitaires. Se situant à mi-chemin entre les blogues et la messagerie instantanée, les micros-blogues sont perçus comme des outils de réseautage social (Murphy, 2008). Plus que de simples tableaux d'affichage, ce sont des outils de communication puissants et pratiques aussi bien pour les bibliothèques que pour leurs usagers. Plusieurs bibliothèques américaines à la renommée internationale, par exemple la Lunar and Planetary Institute Library7, au Texas et la Northeastern Illinois University Library ${ }^{8}$ à Chicago, s'y sont déjà initiées et les utilisent afin de garder le contact avec les utilisateurs par l'échange des réponses rapides et fréquentes.

\section{Les fils RSS}

Le fil RSS est le format XML de syndication de contenu le plus employé de nos jours. Généralement, on parle : de "syndication de contenu " pour désigner la possibilité de republier sur un site Web du contenu provenant d'un autre site Web. C'est une technique qui permet de rendre visibles sur un site Web $A$ les dernières informations parues sur un site Web B de façon synchrone, puisque la mise à jour des informations sur le site B se fait automatiquement (Poupeau, 2004).

Les fils RSS sont actuellement la technologie du Web 2.0 la plus implantée dans les bibliothèques, en particulier dans les bibliothèques universitaires, tel que le démontrent les études de Linh (2008) et Shoniwa \&
Les sites Wikis servent aussi bien à mettre en commun des informations sur les services, les ressources et les activités qu'à créer une interaction entre les bibliothèques et les usagers.

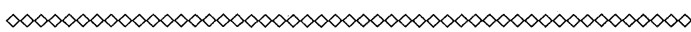

Hall (2007). Ainsi, sur les 152 bibliothèques universitaires britanniques étudiées par Shoniwa \& Hall (2007), 28 utilisaient des fils RSS. Les blogues, implantés dans 17 universités, représentent la deuxième technologie 2.0 la plus implantée.

En matière d'information, de communication et de partage, les fils RSS représentent également une opportunité pour les professionnels d'améliorer leurs prestations de service, entre autres par la souscription aux annonces des nouveautés chez les éditeurs et libraires (Linh, 2008; Shoniwa \& Hall, 2007; Stephens, 2006; Clyde, 2004). Les fils RSS deviennent ainsi un excellent outil de veille au service du développement des collections dans les bibliothèques (Maness, 2006).

\section{Les sites Wikis}

Un site Wiki9 est une page Web ou un ensemble de pages Web qui peuvent être facilement éditées par toute personne y ayant accès, permettant ainsi la rédaction collaborative (Kroski, 2008: 41; Black, 2007: 7 ; Ebersbach et al., 2006). Contrairement aux blogues, les sites Wikis ont généralement une fonction d'archivage qui permet aux versions précédentes d'être examinées, et une fonction "retour en arrière » (rollback) qui permet de les restaurer.

Bien que les sites Wikis soient moins utilisés que les blogues et les fils RSS, ils représentent aussi une opportunité pour les bibliothèques. Cette technologie peut servir comme moyen de communication interne, comme outil de collaboration institutionnelle ou encore comme guide de recherche que les bibliothécaires peuvent utiliser pour répondre aux questions des utilisateurs et connaître leurs rétroactions.

Véritables outils de communication et de collaboration (Frumkin, 2005), les sites.Wikis servent aussi bien à mettre en commun des informations sur les services, les ressources et les activités qu'à créer une interaction entre les bibliothèques et les usagers (Maness, 2006).

\section{Les réseaux sociaux}

Un réseau social incarne une structure dynamique qui se concrétise par l'existence des liens et des rapports sociaux. Les réseaux sociaux sont bien antérieurs à 


\section{Les balados font partie des outils dont disposent les bibliothèques pour varier leurs canaux de diffusion, faire évoluer leurs prestations et, par conséquent, augmenter leur fréquentation.}

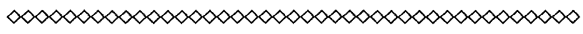

Internet, mais ils ont gagné en popularité notamment avec l'avènement du phénomène Web 2.0. MySpace et Facebook sont des exemples de réseaux sociaux qui bénéficient de l'énorme succès du Web 2.0.

Au même titre que les blogues, les fils RSS ou les sites Wikis, les réseaux sociaux sont une manière pour les bibliothèques d'améliorer leurs moyens de communication et de diffusion, de répondre aux exigences de leurs usagers, d'anticiper leurs besoins et surtout d'attirer les non-usagers. En fait, l'utilisation des outils de réseautage social dans les bibliothèques représente une opportunité à ne pas manquer. Elle serait même vitale pour la survie des bibliothèques et la continuité de leurs prestations (Mathews, 2007).

Très populaires auprès de la communauté académique, les outils de réseautage social ne sont pas sans avoir leurs propres désavantages, notamment lorsqu'il s'agit de protection des informations personnelles des usagers (Sund, 2007). À cet effet, les bibliothèques doivent jouer un rôle important dans l'éducation des usagers sur les dangers de la mise en ligne de leurs renseignements personnels et sur les manières sécuritaires de participer aux communautés en ligne (Farkas, 2007: 123).

\section{Les folksonomies}

La folksonomie est définie comme "un système de classification collaborative et spontanée de contenus Internet, basé sur l'attribution de mots-clés librement choisis par des utilisateurs non spécialistes, qui favorise le partage de ressources et permet d'améliorer la recherche d'informations" (Office québécois de la langue française, 2006).

L'activité individuelle relative à cette classification personnalisée est appelée "étiquetage» ("tagging»). L'activité d'étiquetage a été adoptée de manière progressive au fil des ans et représente aujourd'hui un mode de classification des connaissances spécifique au Web 2.0. Depuis l'intégration du premier système d'étiquetage sur le site del.icio.us en 2003, cette activité a connu d'importantes évolutions et a été adoptée par d'autres sites de partage de contenus, notamment Flickr en 2004, Youtube et Dailymotion en 2005.

Selon Sun (2008) et Spiteri (2007), les folksonomies présentent un réel potentiel qui inciterait les bibliothèques à les incorporer à leurs catalogues en ligne. En raison de leur adaptabilité et souplesse d'utilisation, elles se distinguent des schémas de classification traditionnels jugés rigides, voire inflexibles, par les utilisateurs. En plus de la liberté que les usagers ressentent dans le choix des étiquettes, les folksonomies instaurent un climat de partage qui est à l'origine du renforcement de la dimension sociale prônée par le phénomène du Web 2.0.

\section{Les balados}

Selon l'Office québécois de la langue française (2009), la baladodiffusion est "un mode de diffusion qui permet aux internautes, par l'entremise d'un abonnement à des fils RSS ou équivalents, d'automatiser le téléchargement de contenus radiophoniques, audio ou vidéo, destinés à être transférés sur un baladeur numérique pour une écoute ou un visionnement ultérieurs ». Grâce aux balados, les bibliothèques desservent des utilisateurs éloignés géographiquement et présentant divers profils démographiques (Huffman, 2006). Elles doivent ainsi prévoir des types d'information variés pour accommoder leurs préférences diversifiées. Les balados font partie des outils dont disposent les bibliothèques pour varier leurs canaux de diffusion, faire évoluer leurs prestations et, par conséquent, augmenter leur fréquentation (Huffman, 2006: 15). Linh (2008) identifie trois objectifs derrière l'utilisation des balados dans les bibliothèques :

- rendre visible les contenus et les compétences des bibliothèques et des bibliothécaires;

- produire des capsules d'information pour aider les usagers à se familiariser avec les ressources bibliographiques et l'environnement bibliothéconomique. C'est une autre façon de les former à la maîtrise de l'information;

- guider les usagers dans leurs démarches de recherche sur Internet ou dans les catalogues en ligne en leur permettant d'écouter des balados instructifs sans avoir à lire une documentation imprimée. C'est une façon d'économiser le temps des utilisateurs.

Bien que ces objectifs soient valables, il n'en demeure pas moins que le nombre de bibliothèques utilisant ces technologies reste modeste (Linh, 2008; Shoniwa \& Hall, 2007).

Somme toute, il est important de retenir que les technologies du Web 2.0 sont porteuses de potentiels majeurs pour les bibliothèques. Toutefois, il ne suffit pas d'adopter une technologie et de la laisser fonctionner, encore faut-il en échafauder les bases, suivre son utilisation et prévoir les risques. Une véritable stratégie est ainsi nécessaire dont l'objectif est de définir les objectifs à atteindre, les rapports des professionnels avec cette technologie, les conditions cadres pour réussir l'intégration ainsi que les démarches à entreprendre. 


\section{Conclusion}

Pendant des siècles, on a retrouvé au cœur des missions des bibliothèques les mêmes types d'activités : développer et proposer aux utilisateurs une collection de documents librement accessibles; accomplir un travail de médiation entre les usagers et les ressources informationnelles ; traiter ces ressources avec les mêmes schémas descriptifs traditionnels et les préserver pour les générations futures (Kaufman, 2005). Lavènement des technologies, notamment celles du Web 2.0, suscite des changements dans le mode opératoire des bibliothèques, obligeant ainsi ces dernières à évoluer et à questionner leurs missions traditionnelles.

Développer des collections qui répondent aux besoins des usagers n'est plus une tâche aussi simple. Les aléas que connaît le marché de l'édition, le prix des périodiques, les défis de la gestion des espaces physiques, l'avènement des sources alternatives, l'arrivée de nouvelles technologies de l'information et la complexité de l'information numérique présentent autant de difficultés pour le développement de ces collections (Budd, 2005). D'ailleurs, on se questionne sur l'acception du mot «collection" dans cet environnement informationnel où les bibliothèques sont censées offrir l'accès à des contenus qu'elles peuvent posséder ou sur lesquels elles n'ont pas le contrôle. Quoiqu'il en soit, le recours à certaines technologies, notamment celles du Web 2.0, contribue partiellement à juguler certaines de ces difficultés. Ainsi, grâce aux fils RSS, à titre d'exemple, il est désormais possible pour les bibliothécaires de souscrire aux annonces de nouveautés des éditeurs et des libraires. Il s'agit bien de l'amorce de nouvelles façons de développer les collections et, par conséquent, d'une nouvelle façon pour les bibliothèques d'accomplir leurs missions.

En ce qui concerne l'organisation des collections, il semble que les schémas traditionnels de représentation des connaissances et d'organisation documentaire s'adaptent plus ou moins bien aux ressources électroniques. Les nouveaux schémas d'organisations des ressources numériques, dont les faiblesses ne sont pas négligeables, semblent être plus conviviaux et flexibles (Hudon, 2001). Une autre préférence se fait ressentir pour la recherche libre par mots-clés dans le plein texte et dans les métadonnées, plutôt qu'à l'aide de listes de vedettes-matières et de thésaurus (Hudon, 2001). Dans ce contexte, les recherches ont démontré que les folksonomies, par leur adaptabilité et souplesse d'utilisation, attirent les usagers (Sun, 2008; Spiteri, 2007; Noruzi, 2007). Les folksonomies engendrent un faible "coût " cognitif, en comparaison avec les classifications bibliographiques traditionnelles qui nécessitent un certain consensus (Ertzscheid et Gallezot, 2006). Les bibliothèques, pour mieux répondre aux nouvelles réalités numériques, se doivent de bien comprendre ces différents systèmes d'organisation des connaissances. Elles
Les espaces des bibliothèques peuvent ne plus être utilisés pour stocker et fournir l'accès aux collections, mais peuvent se convertir en lieux de socialisation, de conversation et d'échanges.

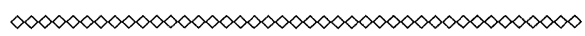

pourront ainsi trouver des solutions hybrides amalgamant systèmes traditionnels contrôlés et nouvelles méthodes libres de catégorisation des contenus.

Pour ce qui est des espaces bibliothéconomiques, il faut reconnaitre que les technologies de l'information, entre autres celles du Web 2.0, remettent sérieusement en question la conception humaine de l'espace, l'utilité de sa fréquentation, voire même sa fonction au sein des bibliothèques. Si les utilisateurs, dans cette conjoncture informationnelle, ne considèrent plus la condition technico-fonctionnelle des espaces bibliothéconomiques, vont-ils pour autant continuer à les fréquenter? Certains chercheurs qui s'intéressent à ces questions n'hésitent pas à parler d'une renaissance des espaces bibliothéconomiques (Eigenbrodt, 2008). Les espaces des bibliothèques peuvent ne plus être utilisés pour stocker et fournir l'accès aux collections, mais peuvent se convertir en lieux de socialisation, de conversation et d'échanges.

En ce qui concerne les services, il semble évident que la philosophie de la bibliothèque 2.0 s'inscrit dans la mouvance enclenchée, voilà des années déjà, par le numérique. Cette mouvance a permis d'évoluer du modèle de la propriété vers un nouveau modèle, celui $\mathrm{du}$ " anywhere, anytime access to information » (Covi \& Cragin, 2004). Ce modèle implique de nouveaux dispositifs de dissémination du savoir, une diversification des canaux et surtout une omniprésence des bibliothèques. Ces dernières doivent réaliser qu'il est temps que leurs contenus possédés ou créés quittent leurs murs pour qu'ils soient disséminés dans d'autres outils, d'autres plateformes, en tout temps et peu importe les lieux où se trouvent les usagers.

De plus, les bibliothèques doivent non seulement répondre à un besoin d'immédiateté, mais doivent également promulguer des services personnalisables, offerts dans l'environnement et selon la logique des usagers. Elles doivent aller au-delà de leur travail de médiation et de communication du savoir pour devenir un espace public de communication. Dans cette optique, les usagers des bibliothèques ne sont plus des consommateurs mais deviennent des collaborateurs, $\mathrm{du}$ moment où ils participent à la collecte, à la création du contenu, à son traitement et à son organisation.

Le Web 2.0, et plus particulièrement sa philosophie et ses outils, représente un réel défi pour les bibliothèques. Ce défi n'est pas insurmontable. Il présente 
au contraire de nouvelles opportunités qui permettront aux bibliothèques et aux bibliothécaires d'accomplir leurs rôles traditionnels différemment. Les bibliothèques n'ont d'autres choix que d'adopter, et de s'approprier, ces nouveaux outils du Web 2.0. Elles pourront ainsi continuer à accomplir leurs fonctions originelles de sélection, d'organisation et de diffusion des connaissances tout en endossant d'autres fonctions au diapason des tendances du Web 2.0. (-)

\section{Sources consultées}

Note : À moins d'indications contraires, toutes les adresses URL étaient valides le 15 juin 2010.

Accart, J-P. (2008). Virturéalité. La bibliothèque sera 2.0... quoique ! Archimag, 33, 4-6.

Albanese, A-R. (2003). Deserted No More. Library Journal 128 (7) : 34-36.

Allard, L. (2007). Émergence des cultures expressives, d'internet au mobile. MédiaMorphoses, $21: 19-25$.

Anderson, P. (2007). What is Web 2.o ? Ideas, Technologies and Implications for Education. Bristol : JISC Technology and Standards Watch. <http://www.jisc.ac.uk/media/documents/ techwatch/tswo7orb.pdf $>$.

Bennett, S. (2005). Righting the Balance, In Library as Place : Rethinking Roles, Rethinking Space. Washington, DC : Council on Library and Information Resources (CLIR pub 129), <http:// www.clir.org/pubs/abstract/pub129abst.html>.

Bernat, L.\& Mesguich, V. (2006). Web 2.0 "5 à 7 " ADBS. Paris, ADBS, <http://www.adbs.fr/evenements/5a7/web-2-o_13-062006.pdf $>$ (page consultée en 2009).

Black, Elizabeth L. (2007). Web 2.0 and library 2.0: What librarians need to know. In Nancy Courtney (Ed.), Library 2.0 and beyond: Innovative technologies and tomorrow's user. Westport, CT : Libraries Unlimited, p. 1-14.

Borgman, C. L. (2003). The invisible library: paradox of the global information structure. Library Trends, 51(4) : 652-674.

Bradley, P. (2007). How to use Web 2.0 in your library. London : Facet Publishing, 224p.

Breeding, M. (2007). We Need to Go Beyond Web 2.o. Computers in Libraries, 27 (5): 22-25.

Brevick, T. (2006). Library 2.0= My library? <http://libipoint5.wordpress.com/2006/>.

Budd, J.M. (2005). The changing academic library operations, culture, environments. Chicago : Association of College and Research Libraries. 336p.

Campbell, J.D. (2006). Changing a cultural icon: The academic library as a virtual destination. EDUCAUSE Review, 41 (1) : 16-30.

Carlson, S. (2001). The Deserted Library: As Students Work Online, Reading Rooms Empty Leading Some Campuses to Add Starbucks. Chronicle of Higher Education. <http://web.archive. org/web/20011109224720/http://www.chronicle.com/free/v48/ i12/12a03501.htm>.

Casey, M. (2006). Born in the Biblioblogsphere. LibraryCrunch. $<$ http://www.librarycrunch.com>.

Casey, M. E. and L.C. Savastinuk (2006). Library 2.0: Service for the Next-Generation Library. Library Journal, 131 (14) : 40-42.

Chowdhury, G., A. Poulter and D. McMenemy (2006). Public library 2.0: Towards a new mission for public libraries as a network of community knowledge. Online Information Review, 30 (4): 454-460.

Clyde, L.A. (2004). Library weblogs. Library Management, 25(4/5) : 183-19o.
Cohen, S. (2006). Library 2.o - Questions and Commentary. <http:// web.archive.org/web/20060110074030/http://www.librarystuff.net/2006/o1/library-20-questions-and-commentary.html> (page consultée en 2009).

Conférence des recteurs et des principaux des universités du Québec (CREPUQ). (2008). L'évolution des bibliothèques universitaires québécoises : un aperçu statistique 1991-2007. $<$ http://www.crepuq.qc.ca/IMG/pdf/Stats_bibliotheques_ CREPUQ_2006-2007_140ctobre2008.pdf >.

Connor, E. (2007). Medical librarian 2.o. Medical Reference Services Quarterly, 26 (1) : 1-15.

Covi, L. M., and M.H. Cragin (2004). Reconfiguring control in library collection development : a conceptual framework for assessing the shift toward electronic collections. Journal of the American Society for Information Science and Technology, 55 (4) : 312-325.

Crawford, W. (2006). Library 2.0 and 'Library 2.0', Cites and Insights, 6, 2. <http://citesandinsights.info/civ6i2.pdf>

Curran, K., M. Murray, and M. Christian (2007). Taking the information to the public through library 2.0. Library Hi Tech, 25 (2) : 288-297.

Curran, K., M. Murray, D.S. Norrby, and M. Christian (2006). Involving the user through library 2.0. New Review of Information Networking, 12 (1-2) : 47-59.

Deshpande, A., and A.R. Jadad (2006). Web 2.0 : Could it Help Move the Health System into the 21st Century. Journal of Men's Health and Gender, 3 (4) : 332-336.

Dowling, T. (2006). LITA blog. <http://litablog.org/ page $/ 6 /$ ? $=$ LIBRARY+2.0 $>$.

Duquet, M. (1993). Glossaire d'écologie fondamentale. Paris : Nathan, $127 \mathrm{p}$.

Eigenbrodt, O. (2008). Espaces sociaux : constitution de l'espace bibliothéconomique par l'activité. In 74 IFLA Council and General Conference, August 10-14, 2008, Berlin, Allemagne. <http:// archive.ifla.org/IV/ifla74/papers/og1-Eigenbrodt-trans-fr.pdf $>$.

Ertzscheid, O., and G. Gallezot (2006). Étude exploratoire des pratiques d'indexation sociale comme une renégociation des espaces documentaires. Vers un nouveau big bang documentaire? In Chartron G. et E. Broudoux, E. (Eds). Document numérique et société. Paris : ADBS. <http://archivesic.ccsd. cnrs.fr/docs/oo/og/16/79/PDF/sdndocsocdefGGOE.pdf >.

Evans, P. (2007). Web 2.0 : la gentillesse d'étrangers. The Boston Consulting Group, Inc. <http://www.bcg.fr/documents/ file8425.pdf $>$.

Farkas, M-G. (2007). Social Software in Libraries: Building Collaboration, Communication, and Community Online. Medford, $\mathrm{NJ}$ : Information Today.

Fichter, D. (2006). Web 2.0, Library 2.0 and Radical Trust : A First Take. <http://library2.usask.ca/ fichter/blog_on_the _ side/2006/04/web-2.html >.

Frumkin, J. (2005). The wiki and the Digital Library. OCLC Systems and Services, $21(1): 18-24$.

Gervais, J. F. (2007). Web 2.o, les internautes au pouvoir: blogs, réseaux sociaux, partage de vidéos, mashups... Paris : Dunod. 224p.

Gordon, R. (2006). NextGen : What Will You Do Today? Library Journal, 5 (11) : 50-53.

Grossman, D. (2007). Internet librarian 2006: 10 years old and going strong. Searcher, 15 (2) : 45-51.

Hill, R., and R. Dunbar (2002). Social Network Size in Humans. Human Nature, 14 (1) : 53-72.

Holmberg, K., I. Huvila, M. Kronqvist-Berg, and G. Widén-Wulff (2009). What is Library 2.0 ? Journal of Documentation, 65 (4) : 668-681.

Houghton, S. (2005). Library 2.0 Discussion : Michael Squared. LibrarianInBlack. <http://librarianinblack.typepad.com/librarianinblack/2005/12/library_20_disc.html>. 
Hudon, M. (2001). Structuration du savoir et organisation des collections dans les répertoires du Web. Bulletin des Bibliothèques de France, 46 (1) : 57-62. <http://bbf.enssib.fr/consulter/ bbf-2001-01-0057-005>.

Huffman, K. (2006). Web 2.0 : beyond the concept practical ways to implement RSS, podcasts and Wikis. Education Libraries, 29 (1) : 12-19.

I-expo 2007. (2007). Les nouvelles valeurs de l'information à l'heure du Web 2.o, Documentaliste-Sciences de l'information, 3 (44): 249-255.

Ibnlkhayat, N. (2005). Marketing des systèmes et services d'information et de documentation - Traité pour l'enseignement et la pratique du marketing de l'information, Québec: Presses de l'Université du Québec (Gestion de l'information). 419p.

Japon. Ministry of Internal Affairs and Communication (MIC). (2006). White paper on Information and Communications in Japan 2006, Chapter 1 : Ubiquitous Economy. <http://www. soumu.go.jp/joho_tsusin/eng/whitepaper.html >.

Kaufman, P. (2005). Role and Mission of Academic Libraries : Present and Future. Japanese Association of Private University Libraries, Kansai University, Osaka, Japan. <https ://www. ideals.uiuc.edu/bitstream/handle/2142/123/Japan \%202005.ppt. pdf ? sequence $=5>$.

Kroski, E. (2007). Folksonomies and user-based tagging. In Courtney, N. (Ed.). Library 2.0 and Beyond: Innovative Technologies and Tomorrow's User. Westwood, CT : Libraries Unlimited, p. 91-103.

Kroski, E. (2008). Web 2.o for Librarians and Information Professionals. New York : Neal-Schuman Publishers. 2oop.

LeMeur, L., and L. Beauvais (2007). Blogs et podcasts. Paris : Dunod. $198 \mathrm{p}$.

Lendrevie, J., and D. Lindon (200o). Mercator. 6 éd. Paris : Dalloz. $788 \mathrm{p}$.

Lévy, P. (2006). Un programme de recherche pour l'économie de l'information. International Journal of Information Sciences for Decision Making, 28. <http://isdm.univ-tln.fr/PDF/isdm28/ isdm28-levy.pdf $>$.

Linh, N.C. (2008). A survey of the Application of Web 2.0 in Australasian University libraries. Library Hi Tech, 26 (4) : 630-653.

Liu, S. (2008). Engaging users : the future of academic library Web sites. College \& Research Libraries, 69 (1) : 6-27.

Maness, J. (2006). Library 2.0 Theory: Web 2.0 and Its Implications for Libraries. Webology, 3 (2), Article 25. <http://webology. $\mathrm{ir} / 2006 / \mathrm{v} 3 \mathrm{n} 2 / \mathrm{a} 25 . \mathrm{html}>$.

Martell, C. (2005). The ubiquitous user : a reexamination of Carlson's deserted library. Portal: Libraries and the Academy, 5 (4): 441-53.

Mathews, B. S. (2007). Online social networking. In Nancy Courtney (Ed.), Library 2.0 and beyond: Innovative technologies and tomorrow's user. Westport, CT : Libraries Unlimited, p. 76-89.

Mercier, S. (2006). Exemples de mises en place de services Web 2.0 dans les bibliothèques. Présentation Powerpoint exposée dans le cadre d'une journée thématique sur les nouveaux usages d'accès et de diffusion de l'information, Novembre 2006, Paris : ADBS. <www.adbs.fr/secteurs/IMG/ppt/merciero61106. ppt>.

Miller, P. \& Chad, K. (2005). Do libraries matter? - The rise of Library 2.o, Talis. <http://www.talis.com/applications/downloads/white_papers/DoLibrariesMatter.pdf $>$.

Mossman, K. (2006). Serving the Niche : Viewing Libraries through Chris Anderson's «Long Tail» Lens. The entity from which ERIC acquires the content, including journal, organization, and conference names, or by means of online submission from the author. Library Journal, 31 (12) : 38-40.

Murphy, J. (2008). Micro-Blogging for Science and Technology Libraries. Science \& Technology Libraries, 28 (4) : 375-378.
Nemec-Poncik, M. (2007). La Web 2 économie s'emballe. Lemondeinformatique. $<\mathrm{http}: / / \mathrm{www}$.lemondeinformatique.fr/ dossiers/lire-la-web-2-economie-s-emballe-54-page-1.html >.

Noruzi, A. (2007). Folksonomies: Why do we need controlled vocabulary? Webology, 4(2). <http://eprints.rclis.org/11287/1/ Folksonomies-_Why_do_we_need_controlled_vocabulary. pdf $>$.

O'Reilly, T. (2007). What Is Web 2.0 : Design Patterns and Business Models for the Next Generation of Software. <http://papers. ssrn.com/sol3/Delivery.cfm/SSRN_ID10o8839_code785949. pdf ?abstractid $=1008839 \&$ mirid $=1>$.

OCDE. (2006a). OECD Information Technology Outlook 2006. Paris : OECD. <http://www.oecd.org/sti/ito>.

OCDE. (2006b). OECD-Italian Minister for Innovation and Technologies Conference, the Future Digital Economy: Digital Content Creation, Distribution and Access, Rome, Italy, 30-31 January. $<$ http://www.oecd.org/sti/digitalcontent/conference>.

Office québécois de la langue française (OQLF). (s.d.). Grand dictionnaire terminologique. $<$ http://www.granddictionnaire. $\mathrm{com} />$.

Quoniam, L., and C. Boutet (2008). Web 2.0, la révolution connectique. Document Numérique, 11 (1-2) : 133-143.

Plutchak, S. (2006). Librarian 5.o. <http://tscott.typepad.com/ tsp/2005/12/librarian_50.html $>$.

Poupeau, G. (2004). L'édition électronique change tout et rien. Dépasser les promesses de l'édition électronique. Le Médiéviste et l'ordinateur, $4 .<\mathrm{http}: / /$ lemo.irht.cnrs.fr/43/43-03.htm>.

Ross, L., P. Sennyey (2008). The Library is Dead, Long Live the Library! The Practice of Academic Librarianship and the Digital Revolution. The Journal of Academic Librarianship, 34 (2) : 145-152.

Salaün, J-M, et F. Muet (2001). Stratégie marketing des services d'information, Paris : Cercle De La Librairie. 221p.

Shoniwa, P., and H. Hall (2008). Library 2.0 and UK academic libraries : drivers and impacts. New Review of Information Networking, 14 (2) : 69-79.

Spiteri, L. (2007). Structure and form of folksonomy tags : The road to the public library catalogue. Webology, $4(2)$. <http://www. webology.ir/2007/v4n2/a41.html >.

Stephens, M. (2006). Exploring Web 2.0 and libraries. Library Technology Reports, 42 (4) : 8-14.

Stephens, M. (2007). Participation in a 2.0. World Library Technology Reports, 43(5), 9.

Sun, B. D. (2008). Folksonomy and Health Information Access : How can Social Bookmarking Assist Seekers of Online Medical Information? Journal of Hospital Librarianship, 8 (1): 119-126.

Sund, C. (2007). Towards an international road-map for cybersecurity. Online Information Review, 31 (5) : 566-582.

Surowiecki, J. (2004). The Wisdom of Crowds. New York: Doubleday. $296 \mathrm{p}$.

Topper, E. F. (2007). What's new in libraries Social networking in libraries. New Library World, 108 (7-8) : 378-380.

Weise, F. (2004). Being there : the library as place. Journal of the Medical Library Association, 92 (1) : 6-13.

Wilson, T.D. (1998). Redesigning the University Library in the Digital Age. Journal of Documentation, 54 (1) : 15-27.

$\mathrm{Xu}, \mathrm{C}$., F. Ouyang, and H. Chu. (2009). The academic library meets Web 2.0: Applications and implications. Journal of Academic Librarianship, 35 (4) : 324-331. 